\title{
Multiresolution Analysis of EEG Signals
}

\author{
Marta Borowska ${ }^{1}$, Natalia Białobłocka $^{1}$ \\ 1 Department of Materials and Biomedical Engineering, Bialystok University of Technol- \\ ogy, Poland
}

\begin{abstract}
This paper reports on a multiresolution analysis of EEG signals. The dominant frequency components of signals with and without observed epileptic discharges were compared. The study showed that there were significant differences in dominant frequency between the signals with epileptic discharges and the signals without discharges. This gives the ability to identify epilepsy during EEG examination. The frequency of the signals coming from the frontal, central, parietal and occipital channels are similar. Multiresolution analysis can be used to describe the activity of brain waves and to try to predict epileptic seizures, thereby contributing to precise medical diagnoses.
\end{abstract}

\section{Introduction}

Human brain electrical activity can be measured from the scalp in a noninvasive way by means of electroencephalography. Due to the conductive properties of biological tissue, this activity reflects the effect of proximal and distal sources of synchronized neuronal activities. The electroencephalogram (EEG) is one of the techniques used in clinical neurophysiology to access information about the condition of the brain. Although EEG recordings have been in clinical use for more than half a century, conventional EEG analysis relies mostly on visual inspection and pattern recognition. Even while this methodology is quite useful, the visual inspection of the EEG is subjective and hardly allows for any systematization. In order to overcome this obstacle, quantitative EEG analysis (qEEG) introduces objective measures reflecting the characteristics of brain activity, as well as its associated dynamics (Rosenblatt et al., 2014).

There is a need to check various methods of mathematical interpretation of recorded time series. One of them is spectral analysis, which is based on the application of the Fourier transform in order to decompose signals into sinusoidal components with fixed frequencies. The power spectrum gives 
the information about frequencies occurring in signals and the dominant frequency for these signals. Wavelet analysis allows one to analyze time and frequency contents of signals simultaneously. It is achieved by fixing a function called mother wavelet (e.g. Morlet wavelet) and decomposing the signal into shifted and scaled versions of this function. It allows one to precisely distinguish local characteristics of signals. Computing the wavelet power spectrum, one can obtain information about occurring frequencies as well as when these frequencies occur. Multiresolution analysis allows one to visualize details and approximations of the given signal on several levels of accuracy. In this paper, the wavelet power spectrum of EEG signals was computed for all levels.

\section{Multiresolution Analysis}

The main mechanism for information signals in the frequency domain is the Fourier transform, which assigns any element of the set $X$ from the time domain to a specific element of a set $Y$ with the frequency domain. Analysis in the frequency domain (or spectral analysis) may provide an answer to the question of whether a registered EEG signal is a harmonic component of a particular frequency. Data recorded electroencephalogram have the form of discrete samples and are sequences of finite length. Therefore, for the spectral analysis of an EEG sequence, the Discrete Fourier Transform (DFT) (Mallat, 1989) should be used:

$$
X(\omega)=\sum_{-\infty}^{\infty} x(n) \cdot e^{-j 2 \pi \frac{\omega}{\omega_{s}} n}
$$

where: $X(\omega)$ - Fourier Transform, $x(n)$ - input signal, $\omega$ - pulsation, $\omega_{s}-$ sampling frequency, $n$ - number of samples.

The power spectrum or power spectral density function:

$$
P(\omega)=|X(\omega)|^{2}
$$

gives a measure of the part to the signal made by each of its sine wave components.

The Fourier transform is not suitable for the analysis of non-stationary signals, or processes of transition characteristics. It uses the sinusoidal carriers that are compact in the frequency domain but not in time domain. For cases in which non-stationary signal analysis is necessary, the windowed Fourier transform or wavelet analysis can be used (Klonowski, 1997). 
One of the most modern approaches to the analysis of time - frequency is wavelet analysis (Gentile et al., 2003). Wavelet representation signal is a function of two variables, which is a linear combination of scalable and shifting function that is Mother Wavelet $\psi$ (Pinsky, 2002).

Wavelet Transform represents a signal in the form of individual short timelines (Lou et al., 2004; Mallat, 1999):

$$
g_{a, b}(t)=\frac{1}{\sqrt{a}} g\left(\frac{t-b}{a}\right),
$$

where: $g_{a, b}(t)$ - analyzing wavelet, $g(t)$ - function parent, $a$ - scale factor, $b$ - time shift.

Continuous Wavelet Transform (CWT) is defined as follows (Pinsky, 2002):

$$
C W T(a, b)=\frac{1}{\sqrt{a}} \int_{-\infty}^{+\infty} x(t) * g\left(\frac{t-b}{a}\right) .
$$

This equation represents the filtered signal analyzed by the signal analyzer, which is scaled in the time domain coefficients. This signal is "stretched" to $a>1$, and "compressed" for $a<1$.

Continuous wavelet transform contains a lot of redundant information. As a result of time-sample and scale factor, the number of the wavelet coefficient can be chosen by substituting $b=n 2^{-m}, a=2^{-m}$, where $m$ is resolution and $n$ is discrete shift. The resulting series of Discrete Wavelet Transform is a wavelet DWT (Hu et al., 2007; Lou et al., 2004).

DWT can be described by the formula (Gawędzki et al., 2015):

$$
g_{m, k}(t)=\frac{1}{\sqrt{2^{m}}} g\left(\frac{t-k * 2^{m}}{2^{m}}\right) .
$$

The wavelet spectrum is obtained by time integration of the continuous wavelet transform (Strambi et al., 2004). Kronland-Martinet et al. (1987) developed an algorithm that allows using the wavelets to provide time frequency representation of the signal. The listing of the program in Matlab implementing this procedure can be found in Muthuswamy et al. (1998).

In the Discrete Wavelet Transformation process, the signal passed through two filters (one for high-frequency and other low-frequency) is divided into so-called approximation and detail. Approximation is subjected to further divisions on the approximation and detail. Details are not subject to further divisions (Stark, 2005).

The main concept associated with the wavelet transform is the scale and the associated different levels of transformation, leading to the so-called 
multiresolution. The lowest level of the scale enables details, such as instantaneous change signals, to be captured. These details can enable the exact scale to be detailed (Lee et al., 2011).

A multiresolution analysis (MRA) was initiated by Mallat (1987). This approach to the analysis of signals consists of a multi-level representation of the signal. Multiresolution analysis is rooted in the fact that a signal is represented as the sum of approximations and details. On each subsequent level, approximations are divided into approximation and details. The approximation does not change with the next iteration.

A multiresolution analysis of $L_{2}(\mathbb{R})$ is a collection of subsets of $L_{2}(\mathbb{R})$ such that:

1. $\ldots \subset V_{-1} \subset V_{0} \subset V_{1} \subset \ldots$,

that satisfies certain self-similarity relations in time/space and scale/frequency, as well as completeness and regularity relations;

2. $\bigcup_{j \in \mathbb{Z}} V_{j}=L_{2}(\mathbb{R})$, closing all spaces $V_{j}$ gives a space of finite energy $L_{2}(\mathbb{R})$;

3. $\bigcap_{j \in \mathbb{Z}} V_{j}=\{0\}$, there is no signal approximating function, which belongs also to the space of all resolutions;

4. There are many sequence spaces $V_{j}$, which comply with conditions $1-3$, $f \in V_{j} \Leftrightarrow f\left(2^{-j \bullet}\right) \in V_{0}$,

all spaces $V_{j}$ are scaled variations of space $V_{0}$ relative to the total displacements;

5. $f \in V_{0} \Leftrightarrow f(\cdot-m) \in V_{0}$ for all $m \in \mathbb{Z}$;

6. Function $\Phi$ is a scaling function or father wavelet. $\Phi \in V_{0}$, such that $\{\Phi(\cdot-m)\}_{m \in \mathbb{Z}}$ is ortagonal base in $V_{0}$.

Generally, the MRA proposes that the scaling function has a significant role in the piecewise approximation of the continuous function $f(t)$ and depends on the scaling index. Note that the MRA is not unique and relies on the selection of the mother wavelet function. The selection of the mother wavelet and scaling function is application-dependent; therefore, no specific selection of the mother wavelet and scaling function can be employed for all applications with the desired results (Ravanfar et al., 2015).

\section{Biomedical Application of Multiresolution Analysis}

Multiresolution analysis is widely applied to solve various problems including data compression, biomedical signal analysis, feature extraction, noise elimination and so on. Gandhi et al. (2011) constructed features 
of EEG signals using MRA analysis. For this purpose, they selected energy, entropy, and standard deviation as parameters for further analysis. They found the most suitable candidate among the wavelet coefficients for accurate classification of the EEG signals. Subha et al. (2010) used different signal processing methods: frequency domain, time - frequency and non-linear techniques to extract the hidden information from normal EEG signals with different events on them. Mirzaei et al. (2010) detected epileptic seizures applying a discrete wavelet-spectral entropy. The EEG signal was decomposed by discrete wavelet transform into its sub-bands. They concluded that this method can discriminate between ictal and healthy subjects of the alpha sub-band. Guo et al. (2010) presented a method for automatic epileptic seizure detection. They used approximate entropy features derived from multiresolution analysis, combined with an artificial neural network, to classify the EEG signals regarding the existence or absence of seizure.

Pal et al. (2010) employed the multiresolution wavelet transform for detection and evaluation of the QRS complex. The test result showed an over $99 \%$ true detection rate for $\mathrm{R}$ peak and base accuracy over $97 \%$, 96\%, 95\%, 98\% for heart rate, $\mathrm{P}$ wave, QRS complex and $\mathrm{T}$ wave, respectively. Banerjee et al. (2012) used the discrete wavelet transform to clear out noise of ECG signals and detect R-peaks by adapting the multiresolution approach. Kumar et al. (2015) explored a method for ECG signal compression based on multiresolution analysis. The results showed that the proposed method achieves a high compression ratio at relatively low distortion.

The method proposed by Subasi (2012) automatically classifies the EMG signals as normal, neurogenic or myopathic. In this research, the usefulness of the different feature extraction methods based on MRI analysis for describing MUP morphology was investigated. Kitlas et al. (2010) presented results of synchronization measures: power spectrum, coherence measure, wavelet power spectrum and wavelet coherence measure for patients with primary dysmenorrhea, uterine myomas, and endometriosis and for a healthy woman. They used multiresolution analysis to choose the appropriate frequency level as the base for computation of the wavelet coherence function. Ali et al. (2016) studied voice disorders that are associated with irregular vibrations of vocal folds. They suggested a multiband approach based on a three-level discrete wavelet transformation. In each band, the fractal dimension (FD) of the estimated power spectrum was estimated. The experiments indicated a significant difference in the spectrum of a normal and pathological subject. 


\section{Application of Multiresolution Analysis for EEG Signals}

The EEGs used in this study, which have been analyzed using MRA analysis, come from PhysioNet. This website offers access to collections of recorded physiologic signals (PhysioBank) (Goldberger et al., 2000). The CHB-MIT database, collected at the Children's Hospital Boston, consists of EEG recordings from pediatric subjects with intractable seizures (Shoeb, 2009). The sampling frequency was $256 \mathrm{~Hz}$. The International 1020 system of EEG electrode positions was used. The signal recording time is about $60 \mathrm{~min}$. Next, fragments of signals were selected in such a way that the length of each analyzed signal was 2048 samples. In this paper, 20 samples containing discharge - group A - and 20 samples without epileptic discharges - group B - were compared. Sharp waves, with approximately $90 \%$ of cases occurring in the temporal region, are associated with a history of seizures. Most of the observed sharp waves were seen in leads F7/F8 (frontal temporal), and T3/T4 (central electrode temporal) (Shoeb, 2009). Multiresolution analysis will be performed on the EEG signals from channels F7-T7, F8-T8, T7-P7, T8-P8, P7-O1, P8-O2. In order to analyze selected EEG recordings, computer programs have been developed in Matlab.

Figure 1 shows a fragment of wavelet decomposition of the EEG signal between epileptic discharges obtained from a patient suffering from epilepsy a) frontal temporal left (F7-T7) levels 1-7 b) frontal temporal right (F8-T8) levels $1-7$.
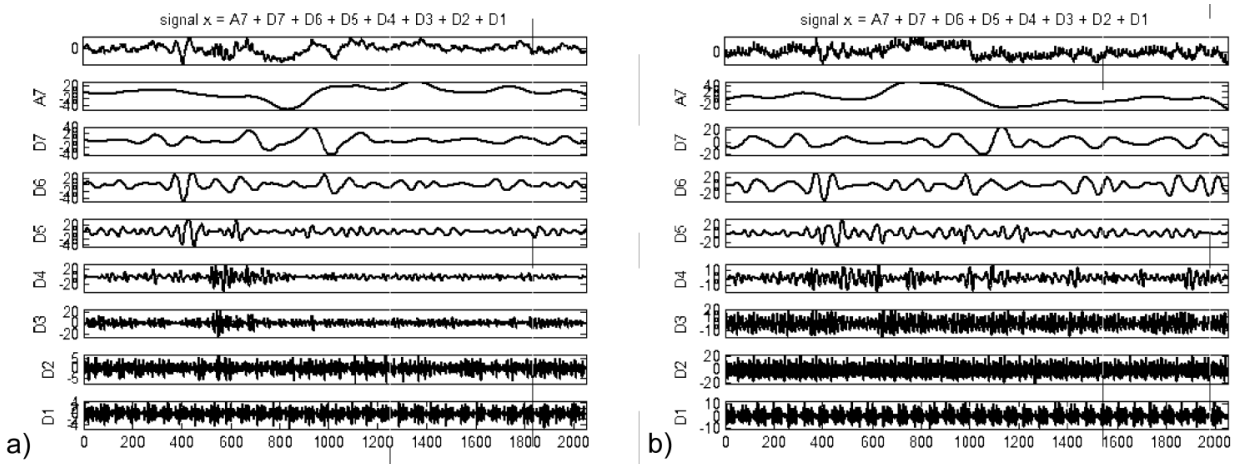

Figure 1. Multiresolution analysis of EEG signal between epileptic discharges: D1-D7 details, A7 - approximation from channels a) F7-T7, b) F8-T8

Figure 2 shows the fragment of wavelet decomposition of the EEG signal during epileptic discharges in the channel with a) the left frontal temporal (F7-T7) levels 1-7, b) the right frontal temporal (F8-T8) levels 1-7. 

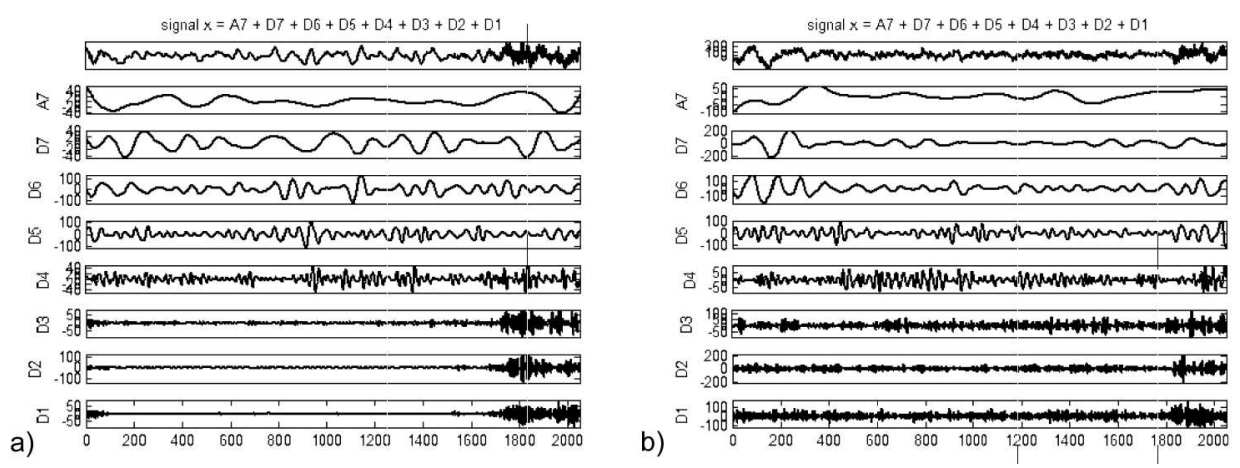

Figure 2. Multi-resolution signal analysis of discharges: D1-D7 details, A7 approximation from channels a) F7-T7, b) F8-T8

After wavelet decomposition of EEG signals, dominant frequency, the frequency at which most signal energy is transmitted, was calculated for all details and approximation from studied channels. Figure 3 shows the example of spectral analysis of an EEG signal: power spectrum using wavelet analysis.
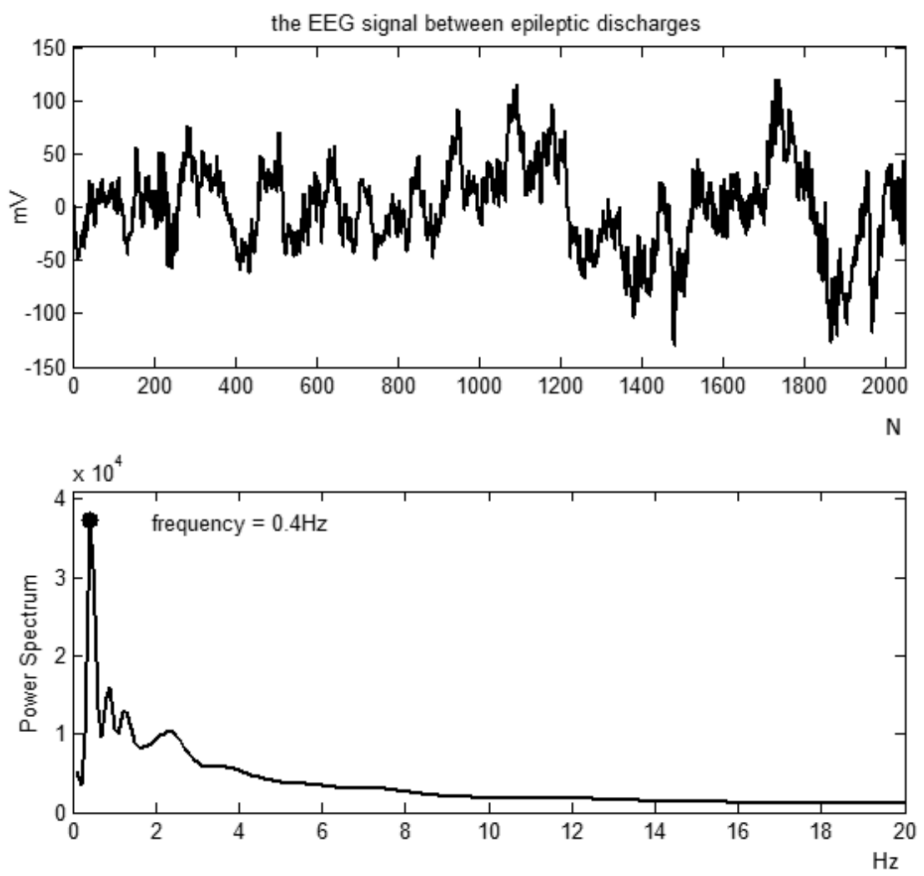

Figure 3. The EEG signal and power spectrum using wavelet transform. Black dot - dominant frequency, the frequency at which most signal energy is transmitted 
Marta Borowska, Natalia Białobłocka

Table 1. The results of dominant frequencies for signals from channels F7-T7 and F8-T8 in studied groups (Mann-Whitney test)

\begin{tabular}{|l|c|c|c|c|}
\hline \multirow{2}{*}{} & \multicolumn{2}{|c|}{ F7-T7 } & \multicolumn{2}{c|}{ F8-T8 } \\
\cline { 2 - 5 } & Group A & Group B & Group A & Group B \\
\hline original & $2.016 \pm 1.371^{* *}$ & $0.600 \pm 0.237^{* *}$ & $1.853 \pm 0.966^{* *}$ & $0.650 \pm 0.271^{* *}$ \\
A7 & $0.579 \pm 0.237$ & $0.531 \pm 0.162$ & $0.679 \pm 0.196$ & $0.600 \pm 0.203$ \\
D1 & $83.116 \pm 1.402$ & $80.525 \pm 4.472$ & $83.074 \pm 2.140^{*}$ & $80.531 \pm 4.510^{*}$ \\
D2 & $37.811 \pm 1.178$ & $38.463 \pm 6.216$ & $37.221 \pm 1.632$ & $38.519 \pm 6.431$ \\
D3 & $20.647 \pm 3.404$ & $21.138 \pm 3.368$ & $20.247 \pm 2.448$ & $21.631 \pm 3.784$ \\
D4 & $10.026 \pm 1.210$ & $10.038 \pm 1.375$ & $10.221 \pm 1.153$ & $10.188 \pm 1.428$ \\
D5 & $4.942 \pm 0.507$ & $4.763 \pm 0.268$ & $4.958 \pm 0.569$ & $4.975 \pm 0.507$ \\
D6 & $2.689 \pm 0.533$ & $2.544 \pm 0.299$ & $2.663 \pm 0.474$ & $2.675 \pm 0.277$ \\
D7 & $1.495 \pm 0.244$ & $1.344 \pm 0.273$ & $1.495 \pm 0.303$ & $1.306 \pm 0.173$ \\
\hline
\end{tabular}

$* p<0.05, * * p<0.001$

Table 2. The results of dominant frequencies for signals from channels T7-P7 and T8-P8 in studied groups (Mann-Whitney test)

\begin{tabular}{|l|c|c|c|c|}
\hline \multirow{2}{*}{} & \multicolumn{2}{|c|}{ T7-P7 } & \multicolumn{2}{c|}{ T8-P8 } \\
\cline { 2 - 5 } & Group A & Group B & Group A & Group B \\
\hline original & $1.900 \pm 1.587^{* *}$ & $0.669 \pm 0.320^{* *}$ & $2.084 \pm 1.349^{* *}$ & $0.606 \pm 0.302^{* *}$ \\
A7 & $0.547 \pm 0.135$ & $0.538 \pm 0.182$ & $0.679 \pm 0.220^{*}$ & $0.519 \pm 0.194^{*}$ \\
D1 & $83.126 \pm 1.346^{* *}$ & $79.581 \pm 4.348^{* *}$ & $83.863 \pm 1.427^{* *}$ & $81.306 \pm 2.729^{* *}$ \\
D2 & $38.211 \pm 0.943$ & $38.438 \pm 6.387$ & $37.842 \pm 1.486$ & $38.169 \pm 2.509$ \\
D3 & $21.116 \pm 3.065$ & $20.469 \pm 3.112$ & $21.305 \pm 3.141$ & $21.781 \pm 3.683$ \\
D4 & $9.795 \pm 1.217$ & $10.444 \pm 2.013$ & $9.632 \pm 0.852$ & $10.275 \pm 1.611$ \\
D5 & $5.137 \pm 0.706$ & $4.994 \pm 0.623$ & $4.916 \pm 0.609$ & $4.838 \pm 0.697$ \\
D6 & $2.547 \pm 0.399$ & $2.563 \pm 0.324$ & $2.653 \pm 0.525$ & $2.438 \pm 0.236$ \\
D7 & $1.405 \pm 0.212$ & $1.350 \pm 0.183$ & $1.547 \pm 0.284^{*}$ & $1.306 \pm 0.208^{*}$ \\
\hline
\end{tabular}

* $p<0.05, * * p<0.001$

The values of dominant frequencies of original signals ranged from $0.3 \mathrm{~Hz}$ to $6.9 \mathrm{~Hz}$ for wavelet analysis. The values of dominant frequencies for these six channels are very similar. Statistical analysis was performed by means of the non-parametric Mann-Whitney test. A significant increase $(p<0.05)$ of dominant frequency of EEG was found in patients with epileptic discharges (group A) (Table 1, Table 2, and Table 3). The levels of details of decomposition were characterized by a decreasing difference between the mean 
Table 3. The results of dominant frequencies for signals from channels P7-O1 and P8-O2 in studied groups (Mann-Whitney test)

\begin{tabular}{|l|c|c|c|c|}
\hline \multirow{2}{*}{} & \multicolumn{2}{|c|}{ P7-O1 } & \multicolumn{2}{c|}{ P8-O2 } \\
\cline { 2 - 5 } & Group A & Group B & Group A & Group B \\
\hline original & $2.321 \pm 1.611^{* *}$ & $0.619 \pm 0.269^{* *}$ & $2.300 \pm 1.538^{* *}$ & $0.619 \pm 0.232^{* *}$ \\
A7 & $0.647 \pm 0.217$ & $0.575 \pm 0.198$ & $0.616 \pm 0.192$ & $0.506 \pm 0.112$ \\
D1 & $82.489 \pm 1.828^{*}$ & $80.250 \pm 3.568^{*}$ & $83.426 \pm 3.228^{*}$ & $80.100 \pm 4.256^{*}$ \\
D2 & $37.168 \pm 1.818$ & $38.825 \pm 3.214$ & $37.884 \pm 1.471$ & $36.744 \pm 3.148$ \\
D3 & $20.816 \pm 3.281$ & $20.938 \pm 3.637$ & $21.121 \pm 3.197$ & $20.100 \pm 3.292$ \\
D4 & $9.700 \pm 0.906$ & $9.869 \pm 1.374$ & $10.000 \pm 1.422$ & $10.038 \pm 1.559$ \\
D5 & $5.011 \pm 0.661$ & $4.863 \pm 0.413$ & $4.958 \pm 0.707$ & $4.794 \pm 0.349$ \\
D6 & $2.789 \pm 0.516$ & $2.519 \pm 0.331$ & $2.684 \pm 0.497$ & $2.431 \pm 0.311$ \\
D7 & $1.537 \pm 0.304^{*}$ & $1.300 \pm 0.222^{*}$ & $1.532 \pm 0.291^{*}$ & $1.306 \pm 0.224^{*}$ \\
\hline
\end{tabular}

$* p<0.05, * * p<0.001$

dominant frequencies. Significant differences were for details D1 and D7. We did not find statistically significant differences between results obtained by means these six different channels of wavelet analysis (Table 1, Table 2, and Table 3). The standard deviation data indicate that we're more focused around the mean of the results of group B.

\section{Conclusions}

Wavelet analysis allows one to simultaneously show time and frequency signal properties and their distribution. Multiresolution analysis gives an opportunity to see approximations and details of a signal and select the appropriate level of detail for further research. The advantage of this type of analysis is high accuracy when considering the local characteristics of the signals.

EEG analysis showed significant differences between the dominant frequency of epileptic discharge signals and signals without discharge. This gives one the ability to identify when the EEG showed a seizure. The levels of details of decomposition are characterized by a decreasing difference between the mean dominant frequencies. Significant differences were for D1 and D7, which gives the opportunity for further research into these details. In conclusion, as supported by research presented herein, multiresolution analysis can be used to test the prediction of epileptic seizures, thereby contributing to a precise medical diagnosis. 


\section{Acknowledgments}

This paper was supported by statutory funds S/WM/1/2014 from the Bialystok University of Technology.

\section{R E F E R E N C E S}

Ali, Z., Elamvazuthi, I., Alsulaiman, M., \& Muhammad, G. (2016). Detection of Voice Pathology using Fractal Dimension in a Multiresolution Analysis of Normal and Disordered Speech Signals. Journal of Medical Systems, 40(1), $1-10$.

Banerjee, S., Gupta, R., \& Mitra, M. (2012). Delineation of ECG characteristic features using multiresolution wavelet analysis method. Measurement, 45(3), 474-487.

Gandhi, T., Panigrahi, B. K., \& Anand, S. (2011). A comparative study of wavelet families for EEG signal classification. Neurocomputing, 74(17), 3051-3057.

Gawędzki, W., Socha, M., \& Sławik, P. (2015). Dekompozycja sygnałów EEG w dziedzinie czasu przy zastosowaniu transformacji Hilberta-Huanga HHT. Przeglad Elektrotechniczny, 91, 33-36.

Gentile, A., \& Messina, A. (2003). On the continuous wavelet transforms applied to discrete vibrational data for detecting open cracks in damaged beams. International Journal of Solids and Structures, 40(2), 295-315.

Goldberger, A. L., Amaral, L. A., Glass, L., Hausdorff, J. M., Ivanov, P. C., Mark, R. G., Mietus, J. E., et al. (2000). Physiobank, physiotoolkit, and physionet. Components of a new research resource for complex physiologic signals. Circulation, 101(23), e215-e220.

Guo, L., Rivero, D., \& Pazos, A. (2010). Epileptic seizure detection using multiwavelet transform based approximate entropy and artificial neural networks. Journal of Neuroscience Methods, 193(1), 156-163.

Hu, Q., He, Z., Zhang, Z., \& Zi, Y. (2007). Fault diagnosis of rotating machinery based on improved wavelet package transform and SVMs ensemble. Mechanical Systems and Signal Processing, 21(2), 688-705.

Kitlas, A., Oczeretko, E., Laudański, P., \& Laudański, T. (2010, August). Signal processing methods in the analysis of the uterine contractility. Paper presented at the 6th World Congress of Biomechanics (WCB 2010), Singapore.

Klonowski, W. (1997). Zastosowanie teorii układów nieliniowych do analizy sygnału EEG. X Krajowa Konferencja Naukowa: Biocybernetyka i Inżynieria Biomedyczna. Materialy naukowe, 2, 590-594.

Kronland-Martinet, R., Morlet, J., \& Grossmann, A. (1987). Analysis of sound patterns through wavelet transforms. International Journal of Pattern Recognition and Artificial Intelligence, 1(02), 273-302.

Kumar, R., \& Kumar, A. (2015). ECG signal compression algorithm based on jointmultiresolution analysis $(J-M R A)$. Paper presented at the 2nd International Conference on Electronics and Communication Systems (ICECS). 
Lee, S. H., \& An, J. (2011). Quantitative EEG evaluation of multimedia intervention program for $A D H D$ children. Paper presented at the Proceedings of the 4th International Symposium on Applied Sciences in Biomedical and Communication Technologies.

Lou, X., \& Loparo, K. A. (2004). Bearing fault diagnosis based on wavelet transform and fuzzy inference. Mechanical Systems and Signal Processing, 18(5), 10771095.

Mallat, S. G. (1987). A Theory for Multiresolution Signal Decomposition: The Wavelet Representation (University of Pennsylvania, Department of Computer and Information Science Technical Report No. MS-CIS-87-22).

Mallat, S. G. (1989). A Theory for Multiresolution Signal Decomposition: The Wavelet Representation. IEEE Transactions on Pattern Analysis and Machine Intelligence, 11(7), 674-693.

Mallat, S. G. (1999). A wavelet tour of signal processing. Academic press.

Mirzaei, A., Ayatollahi, A., Gifani, P., \& Salehi, L. (2010). EEG analysis based on wavelet-spectral entropy for epileptic seizures detection. Paper presented at the 3rd International Conference on Biomedical Engineering and Informatics.

Muthuswamy, J., \& Thakor, N. V. (1998). Spectral analysis methods for neurological signals. Journal of Neuroscience Methods, 83(1), 1-14.

Pal, S., \& Mitra, M. (2010). Detection of ECG characteristic points using multiresolution wavelet analysis based selective coefficient method. Measurement, $43(2), 255-261$.

Pinsky, M. A. (2002). Introduction to Fourier analysis and wavelets. Graduate Studies in Mathematics, Volume 102, American Mathematical Society.

Ravanfar, S. A., Razak, H. A., Ismail, Z., \& Monajemi, H. (2015). An improved method of parameter identification and damage detection in beam structures under flexural vibration using wavelet multi-resolution analysis. Sensors, 15(9), 22750-22775.

Rosenblatt, M., Figliola, A., Paccosi, G., Serrano, E., \& Rosso, O. A. (2014). A Quantitative Analysis of an EEG Epileptic Record Based on MultiresolutionWavelet Coefficients. Entropy, 16(11), 5976-6005.

Shoeb, A. H. (2009). Application of machine learning to epileptic seizure onset detection and treatment. Massachusetts Institute of Technology.

Stark, H.-G. (2005). Wavelets and signal processing: an application-based introduction. Springer Science \& Business Media.

Strambi, S.-k., Rossi, B., De Michele, G., \& Sello, S. (2004). Effect of medication in Parkinson's disease: a wavelet analysis of EMG signals. Medical Engineering \& Physics, 26(4), 279-290.

Subasi, A. (2012). Classification of EMG signals using combined features and soft computing techniques. Applied Soft Computing, 12(8), 2188-2198.

Subha, D. P., Joseph, P. K., Acharya, R., \& Lim, C. M. (2010). EEG signal analysis: a survey. Journal of Medical Systems, 34(2), 195-212. 